\title{
Changes in proprioceptive weighting during quiet standing in women with early and established knee osteoarthritis compared to healthy controls
}

\author{
Armaghan Mahmoudian ${ }^{\mathrm{a}}$, Jaap H. van Dieen ${ }^{\mathrm{b}}$, Isabel A.C. Baert ${ }^{\mathrm{c}}$, Ilse Jonkers ${ }^{\mathrm{d}}$, \\ Sjoerd M. Bruijn ${ }^{\mathrm{b}, \mathrm{e}}$, Frank P. Luyten ${ }^{\mathrm{f}}$, Gert S. Faber ${ }^{\mathrm{b}}$, Sabine M.P. Verschueren ${ }^{\mathrm{a}, *}$ \\ ${ }^{a}$ Department of Rehabilitation Sciences, Faculty of Kinesiology and Rehabilitation Sciences, KU Leuven, Belgium \\ ${ }^{\mathrm{b}}$ MOVE Research Institute Amsterdam, Faculty of Human Movement Sciences, VU University Amsterdam, The Netherlands \\ ${ }^{\mathrm{c}}$ Department of Health Care, Artesis University College of Antwerp, Belgium \\ ${ }^{\mathrm{d}}$ Department of Kinesiology, Faculty of Kinesiology and Rehabilitation Sciences, KU Leuven, Belgium \\ ${ }^{\mathrm{e}}$ Department of Orthopedics, First Affiliated Hospital of Fujian Medical University, Fuzhou, Fujian, China \\ ${ }^{\mathrm{f}}$ Department of Development \& Regeneration, Skeletal Biology and Engineering Research Center, KU Leuven, Leuven, Belgium
}

\section{A R T I C L E I N F O}

\section{Article history:}

Received 15 June 2015

Received in revised form 17 November 2015

Accepted 3 December 2015

\section{Keywords:}

Knee osteoarthritis

Postural control

Proprioception

Vibration

\begin{abstract}
A B S T R A C T
Objectives: Knee osteoarthritis (OA) is highly prevalent in people above the age of 60, and is typically associated with pain, stiffness, muscle weakness and proprioceptive deficits. Muscle-tendon vibration has been used to assess the spatial reweighting of proprioceptive input during standing. The current study aimed to investigate whether weighting of proprioceptive input is altered in patients with early and established knee OA compared to asymptomatic controls.

Methods: The upright posture of 27 participants with early OA, 26 with established OA, and 27 asymptomatic controls was perturbed by vibrating (frequency: $70 \mathrm{~Hz}$ and amplitude: approximately $0.5 \mathrm{~mm}$ ) ankle muscles (i.e. tibialis anterior and triceps surae) and knee muscles (vastus medialis). Center of pressure displacements of the participants were recorded using a force plate.

Results: Both patients with early and established OA were more sensitive to triceps surae vibration compared to their healthy peers $(P<0.01$ for both). No such difference was found for the vibration of tibialis anterior or vastus medialis muscles between patients with knee OA and healthy controls. Conclusions: These results suggest that the early stages of knee OA may already lead to reweighting of proprioceptive information, suggesting more reliance on ankle proprioceptive input for postural control.
\end{abstract} (c) 2015 Elsevier B.V. All rights reserved.

\section{Introduction}

Maintaining upright posture requires the central nervous system (CNS) to accurately observe the instantaneous state of the body relative to the environment. The body state is observable through a range of sensory inputs arising from vestibular, visual, and somatosensory systems [1]. The proprioceptive input from the

\footnotetext{
* Corresponding author at: Department of Rehabilitation Sciences, KU Leuven., Tervuursevest 101, 3001 Heverlee, Belgium. Tel.: +32 163291 70;

fax: +3216329192.

E-mail addresses: armaghan.mahmoudian@gmail.com (A. Mahmoudian), j.van.dieen@vu.nl (J.H. van Dieen), isabel.baert@uantwerpen.be (Isabel A.C. Baert), ilse.jonkers@faber.kuleuven.be (I. Jonkers), s.m.bruijn@gmail.com (S.M. Bruijn), frank.luyten@uzleuven.be (F.P. Luyten), gertfaber.sci@gmail.com (G.S. Faber), sabine.verschueren@faber.kuleuven.be (Sabine M.P. Verschueren).
}

lower limb muscles is crucial in preserving postural stability [2], which implies that impoverished afferent signals from these muscles might compromise postural stability. As an example, subjects with dorsal root ganglionopathy show severe balance impairments, due to absence of lower limb proprioception [3]. Certain conditions such as injury, disease, or aging may negatively affect the quality of input from affected body parts [4]. In such cases, the CNS needs to substitute for the impaired source by using more information from other available sources such as vision or proprioceptive information from other body parts, to maintain a stable posture [5].

Knee osteoarthritis (OA) is highly prevalent in people above the age of 60 and has been associated with proprioceptive deficits [6-8] and postural control deficits $[9,10]$. However, reports of impaired proprioception in knee OA populations have thus far mostly been based on testing conscious perception of posture or movement [6-8], 
while a better understanding of the role of a specific sensory system in postural control might be achieved through bypassing the role of conscious perception in testing [11]. Muscle-tendon vibration has been used to assess the weight allocated to proprioceptive inputs from different body parts [4]. Muscle vibration stimulates the primary afferents of muscle spindles [12] and results in an illusory perception of muscle lengthening [13]. The vibrated muscle is perceived to lengthen, and as a result of this distorted sensory information, a corrective movement is made. The direction of this corrective postural response differs depending on the origin of the distorted information, and the magnitude depends on the weight that the CNS allocates to input from this body part compared to the other sources of information [4]. For instance, in a study on postural weighting of patients with low back pain by Brumagne et al., persons with low back pain showed larger CoP shifts towards posterior direction compared to the healthy individuals when vibration was applied bilaterally on the triceps surae, suggesting more reliance on ankle input [4]. Only one recent study by Shanahan et al. used muscle vibration to assess the proprioceptive weighting (PW) in a group of subjects with severe knee OA (Kellgren and Lawrence grade 3 or 4) [11]. Participants with knee OA were initially perturbed more by triceps surae (TS) than vastus medialis (VM) vibration compared to control subjects [11], from which it was concluded that these participants were unable to compensate the induced and nonveridical sensory signals from the TS by using the information from the VM [11]. To the best of our knowledge, proprioceptive weighting has not yet been studied in the early stage of knee OA. Such understanding might be helpful for development of more purposive preventive or therapeutic strategies.

Proprioceptive deficits associated with knee OA have been considered as a potential cause for observed changes in proprioceptive weighting in this population [11], however, there are no studies on the relationship between PW and proprioceptive accuracy in the population of subjects with knee OA. In the current study we also investigated this relationship by including the proprioceptive accuracy of subjects with early and established knee OA [8].

Consequently, to better understand the progression of proprioceptive impairments with the progression of knee OA, the aim of this study was: (1) to investigate proprioceptive weighting in a group of patients with early knee OA, patients with established knee OA and to compare them with healthy peers; (2) to explore whether the sensitivity of the knee muscle to vibration decreases with increasing severity of knee $\mathrm{OA}$; $(3)$ to explore if there is a relationship between proprioceptive weighting and proprioceptive accuracy in subjects with knee OA.

\section{Materials and methods}

Fifty-two women with medial knee OA and 27 asymptomatic women participated in this study. Participants with knee OA were recruited during their regular visit to a rheumatologist or orthopedic surgeon at the University Hospitals Leuven. Participants in the healthy control group were recruited through social organizations. All participants were informed about the study procedure and signed informed consent forms. The study was approved by the ethical committee for Biomedical Sciences of the $\mathrm{KU}$ Leuven in Belgium prior to testing and was conducted in agreement with the principles of Declaration of Helsinki.

Each participant was referred for a physical exam and bilateral standard anterior-posterior weight-bearing radiographs in fixed flexed position were obtained (Siemens, Siregraph CF, Agfa CR HD5.0 detector $24^{*} 30$ ). Diagnosis and categorization of knee OA were based on the K\&L grading system [14] and a single experienced observer (FPL) graded each radiograph. A magnetic resonance image (MRI) was taken from the (most) affected side of the OA patients, based on radiography, and a random side in the control group, as described by Baert et al. [15].

The standardized Boston-Leeds Osteoarthritis Knee Score (BLOKS) scoring system was used by two separate readers (NN, GVDS) to score structural features in the tibiofemoral joint [16]. On $91 \%$ of all scored items, the two readers had full agreement and disagreements were resolved by consensus.

Participants with knee OA were further sub-classified, into early $(n=27)$ and established $(n=26)$ medial knee OA groups [17]. The inclusion criteria for the early OA group were: presence of knee pain, a K\&L grade 0,1 or $2-$ for the medial compartment, and presence of two of four MRI criteria: (1) $\geq$ BLOKS grade 2 for size cartilage loss, (2) $\geq$ BLOKS grade 2 for percentage full-thickness cartilage loss, (3) signs of meniscal degeneration and (4) $\geq$ BLOKS grade 2 for size of bone marrow lesions (BMLs) in any one compartment.

The classification of participants in the established knee OA group was based on the slightly adjusted American College of Rheumatology (ACR) classification criteria [18], which includes knee pain, age above 50, stiffness less than 30 min and crepitus, combined with structural changes defined as presence of minimum K\&L grade $2+$, indicating a moderate to severe disease severity.

The inclusion criteria for the control group were as follows, K\&L grade 0 or 1 on the radiography of either knee, asymptomatic, no history of knee OA or other pathology involving any lower extremity joints.

\subsection{Clinical assessment}

To assess knee symptoms and function, the Knee Injury and Osteoarthritis Outcome Score (KOOS) (Dutch version) was filled in by all participants. Validity and reliability of the KOOS has been verified for evaluation of short- and long-term symptoms and function in knee OA patients [19,20].

\subsection{Proprioceptive weighting and postural control assessment}

Postural control was assessed using a six-channel force plate (Bertec, Corporation, Ohio, USA). Force plate data were sampled at 1000 samples/s. Participants were asked to comfortably stand barefoot on the force platform with arms crossed in front of the chest and the feet slightly separated. In all trials, vision was occluded by means of a blindfold. Each participant underwent three experimental conditions during which they were instructed to stand still and relaxed. The three conditions were: (1) bilateral vibration of the TS tendons; (2) bilateral vibration of the tibialis anterior (TA) muscle bellies; and (3) bilateral vibration of the VM muscle bellies. Two muscle vibrators (VB100, Dynatronic, Valence, France) were attached over the most proximal part of the tendon of the triceps surae muscles, and vastus medialis muscle belly using straps. The tightness of these straps was subjectively checked with the subject. The activation (frequency of $70 \mathrm{~Hz}$, amplitude of approximately $0.5 \mathrm{~mm}$ ) and deactivation of the vibrators was controlled manually. These characteristics of vibration were chosen to induce the maximal illusory joint movement [21]. Each trial lasted $45 \mathrm{~s}$, during which muscletendon vibration was applied for $15 \mathrm{~s}$, initiated $15 \mathrm{~s}$ after the start of the trial. Data collection continued for $15 \mathrm{~s}$ after the vibration was stopped.

All participants were asked to stop the test whenever they felt discomfort or pain during the test procedure. In case a participant lost her balance and tended to fall, the trial was excluded and repeated. As all subjects participated in the current study fulfilled every test trial without difficulty, we do assume that they did not experience pain related to the test procedures. 


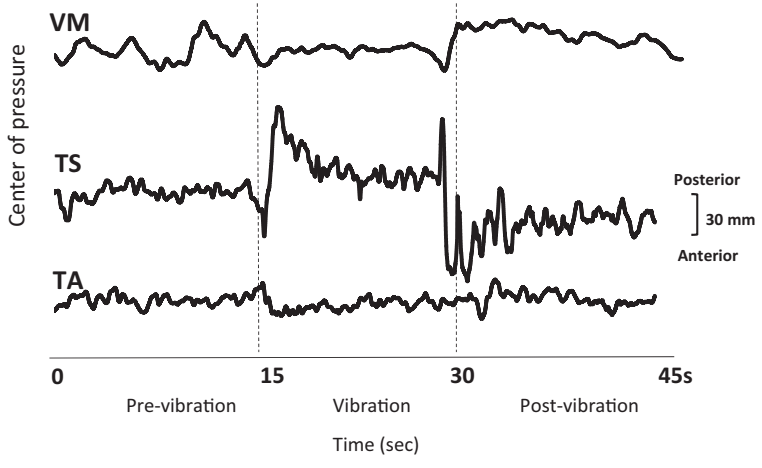

Fig. 1. CoP (anteroposterior) position of a representative participant. Vibration was applied to tibialis anterior (TA), triceps surae (TS), and vastus medialis (VM).

The center of pressure (CoP) position was calculated and averaged over the first $15 \mathrm{~s}$ of the trial (pre-vibration) and during the $15 \mathrm{~s}$ of vibration. The response to muscle vibration was defined and quantified as the difference in mean CoP position before and during vibration (Fig. 1).

Proprioceptive weighting between ankle and knee muscles was calculated as:

$$
\mathrm{PW}_{\mathrm{TA}-\mathrm{VM}}=\frac{\left|\mathrm{TA}_{\text {response }}\right|}{\left|\mathrm{TA}_{\text {response }}\right|+\left|\mathrm{VM}_{\text {response }}\right|},
$$

and

$\mathrm{PW}_{\mathrm{TS}-\mathrm{VM}}=\frac{\left|\mathrm{TS}_{\text {response }}\right|}{\left|\mathrm{TS}_{\text {response }}\right|+\left|\mathrm{VM}_{\text {response }}\right|}$,

where PW stands for proprioceptive weighting.

\subsection{Proprioceptive accuracy}

Proprioceptive accuracy was examined using an active repositioning test [22]. The participant was seated on a chair with knees flexed $\left(90^{\circ}\right.$ flexion, hanging relaxed and unsupported) over the edge of the chair and with the eyes closed. The knee was extended passively from the resting position to one of the three test positions: $70^{\circ}, 45^{\circ}$, and $20^{\circ}$ flexion. This knee angle (criterion angle) was maintained by the participant for $3 \mathrm{~s}$. The knee was then flexed back to the resting position ( $90^{\circ}$ flexion) and relaxed for $3 \mathrm{~s}$. Subsequently, the participant was asked to replicate the test position and hold it for $3 \mathrm{~s}$. After familiarization with the test, each participant performed the tests twice in each of the knee angles in a standardized order. The motion was tracked using an active three dimensional (3D) motion capture system at 100 samples/s (Krypton, Metris), using a previously described protocol [8].
Repositioning error (RE) was defined as the absolute difference between the criterion angles and reproduced angles. Four variables were calculated: mean RE of all six tests together and mean RE for the three different test positions separately.

\section{Statistics}

Descriptive statistics were used to summarize the characteristics of the study population. One-way analyses of variance (ANOVA) (if data were normally distributed and had equal variances) or Kruskal-Wallis tests (if data were not normally distributed or variances were not equal) were used to test for group differences in demographic and clinical characteristics. If indicated, Bonferroni corrected paired t-tests or Wilicoxon tests were used post-hoc in conjunction with the ANOVA's and KruskalWallis tests, respectively.

Differences between groups for: response, recovery, proprioceptive weighting, and repositioning error were tested with general estimating equations (GEEs), with group as factor. For post hoc analysis, pairwise comparisons were used.

To assess associations between proprioceptive weighting and proprioceptive accuracy, Pearson product moment correlation coefficients were used within the total OA group, the early OA and established OA group. All statistical analyses were performed using SPSS 22.0 (SPSS Inc., Chicago, USA), with level of significance set at $P<0.05$

\section{Results}

Participants' characteristics are reported in Table 1. No significant differences were detected between groups in age, height, weight, and BMI. As expected, participants with OA had higher KOOS scores on all subscales but there was no significant difference between the two OA groups regarding any of the KOOS sub-scores.

\subsection{Proprioceptive weighting and postural control assessment}

As can be seen in Fig. 1, vibration of all three muscles resulted in a shift of the CoP, but the direction, in which the CoP shifted, was different between muscles. Vibration of the TS led to a posterior shift of the CoP, while vibration of TA and VM resulted in an anterior shift of the CoP. For all three muscles, a shift of the CoP back towards baseline occurred after termination of the vibration.

In response to TS vibration, the early and established OA groups showed a larger posterior shift of the CoP compared to the controls, but did not differ from each other (Table 2). Vibration of the VM resulted in an anterior shift of the CoP in all three groups, but this response did not differ between groups $(P=0.521)$. Regarding the effect of TA vibration, there was no significant difference between the three groups (Table 2).

Table 1

Participant characteristics and results for tests of differences between groups.

\begin{tabular}{|c|c|c|c|c|c|c|c|}
\hline Characteristics & Control $(n=27)$ & Early $(n=27)$ & Established $(n=26)$ & $P$ & $\begin{array}{l}P \text { (established } \\
\text { vs. control) }\end{array}$ & $\begin{array}{l}P \text { (early vs. } \\
\text { control) }\end{array}$ & $\begin{array}{l}P \text { (early vs. } \\
\text { established) }\end{array}$ \\
\hline Age (years) ${ }^{\mathrm{a}, \mathrm{d}}$ & $64.63(7.6)$ & $66.85(6.5)$ & $66.13(7)$ & 0.471 & & & \\
\hline Weight $(\mathrm{kg})^{\mathrm{a}, \mathrm{d}}$ & $65.08(11.1)$ & $69.72(11.4)$ & $71.46(11.8)$ & 0.076 & & & \\
\hline Height $(m)^{a, c}$ & $1.60(0.1)$ & $1.63(0.1)$ & $1.60(0.1)$ & 0.264 & & & \\
\hline $\operatorname{BMI}\left(\mathrm{kg} / \mathrm{m}^{2}\right)^{\mathrm{a}, \mathrm{d}}$ & $25.23(4)$ & $26.35(4.3)$ & $27.82(4.6)$ & 0.058 & & & \\
\hline KOOS pain score $e^{b, d}$ & $100(2.8)$ & $86.1(27.8)$ & $80.5(33.3)$ & $<0.001^{*}$ & $<0.001^{*}$ & $<0.001^{*}$ & 0.241 \\
\hline KOOS symptoms score ${ }^{\mathrm{b}, \mathrm{d}}$ & $100(8.3)$ & $83.33(33.3)$ & $75(33.3)$ & $<0.001^{*}$ & $<0.001^{*}$ & $<0.001^{*}$ & 0.156 \\
\hline KOOS ADL score ${ }^{b, d}$ & $100(1.5)$ & $88.2(28)$ & $85.2(39.7)$ & $<0.001^{*}$ & $<0.001^{*}$ & $<0.001^{*}$ & 0.256 \\
\hline
\end{tabular}

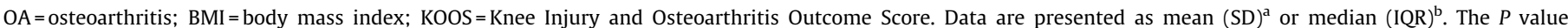
corresponds to an ANOVA ${ }^{c}$, Kruskal-Wallis test (with post hoc tests) ${ }^{\mathrm{d}}$ comparing the three groups.

Significant difference between groups $(P<0.05)$. 
Table 2

Mean values (SD) of CoP displacements during and after muscle vibration, and GEE results with Group (established OA vs. early OA vs. controls) as factor.

\begin{tabular}{|c|c|c|c|c|c|c|c|}
\hline & $\begin{array}{l}\text { Control } \\
(n=27)\end{array}$ & $\begin{array}{l}\text { Early OA } \\
(n=27)\end{array}$ & $\begin{array}{l}\text { Established OA } \\
(n=26)\end{array}$ & $P$ & $\begin{array}{l}P \text { (established } \\
\text { vs. control) }\end{array}$ & $\begin{array}{l}P \text { (early } \\
\text { vs. control) }\end{array}$ & $\begin{array}{l}P \text { (early vs. } \\
\text { established) }\end{array}$ \\
\hline \multicolumn{8}{|l|}{ CoP displacement } \\
\hline Response TA (mm) & $15.35(2.2)$ & $15.11(2.2)$ & $14.6(2.3)$ & 0.99 & & & \\
\hline Response TS (mm) & $-20.44(3.7)$ & $-38.86(3.7)$ & $-36.62(3.7)$ & $0.001^{*}$ & $0.005^{*}$ & $<0.001^{*}$ & 0.484 \\
\hline Response VM (mm) & $1.45(1.6)$ & $3.69(1.6)$ & $4.24(1.8)$ & 0.521 & & & \\
\hline \multicolumn{8}{|l|}{ Proprioceptive weighting } \\
\hline $\mathrm{PW}_{\mathrm{TA}-\mathrm{VM}}$ & $0.71(0.04)$ & $0.70(0.05)$ & $0.70(0.05)$ & 0.963 & & & \\
\hline $\mathrm{PW}_{\mathrm{TS}-\mathrm{VM}}$ & $0.81(0.02)$ & $0.87(0.02)$ & $088(0.02)$ & $0.036^{*}$ & $0.017^{*}$ & $0.049^{*}$ & 0.647 \\
\hline
\end{tabular}

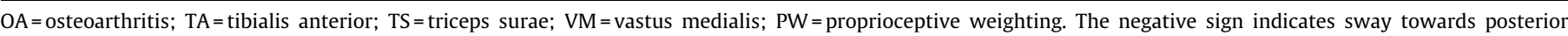
direction. Data are presented as mean (SD).

Significant difference between groups $(P<0.05)$.

Proprioceptive weighting between TS and VM (PW $\left.\mathrm{PW}_{\mathrm{TS}} \mathrm{VM}\right)$ was significantly different between the three groups, showing higher PW ratio's for both groups with early and established knee OA compared to healthy participants (Table 2), but no differences between these groups. On the other hand, proprioceptive weighting between TA and VM ( $\mathrm{PW}_{\mathrm{TA}-\mathrm{VM})}$ was not significantly different between the three groups $(P=0.963)$.

\subsection{Proprioceptive accuracy}

The mean repositioning error values for all three groups are presented in Fig. 2. Proprioceptive accuracy was not significantly different between early $\mathrm{OA}$ and control groups (Fig. 2). The established OA group showed significantly higher RE values compared to the control group $(P=0.003)$ when combining all tests and compared to both the early OA group and the control group $\left(P=0.026\right.$ and $P=0.006$, respectively) for tests in $45^{\circ}$ flexion.

\subsection{Relationship between proprioceptive accuracy and proprioceptive weighting}

Considering patients with early and established knee OA, no significant correlations were found between TS response and RE in any of the testing positions $\left(r_{70}=0.008, P_{70}=0.946 ; r_{45}=-0.105\right.$, $P_{45}=0.355 ;$ and $\left.r_{20}=0.108, P_{20}=0.341\right)$.

\section{Discussion}

The current study investigated the association of proprioceptive impairments with the progression of knee OA by comparing proprioceptive weighting in women with early and established medial knee OA and control participants. Results showed that women with knee OA are more sensitive to vibration of the triceps surae muscle, than vibration of the vastus medialis muscle, compared to healthy controls. Both OA groups included in this study showed an enhanced response to TS muscle vibration, manifested as an increased posterior shift of the CoP compared to the healthy controls. Shanahan et al. also reported increased sensitivity to TS muscle vibration in a group of participants with severe knee OA (with KL grade of 3 or 4) [11]. The present study extended the previous findings by showing that these changes already exist at time of early joint degeneration.

The aforementioned changes in sensitivity to vibration of the TS with knee OA could result from changes in the central processing of this afferent information. It has been established that participants with knee OA suffer from knee joint proprioception deficits [6-8], therefore, the proprioceptive information from the knee might be inadequate or distorted in a way that the CNS cannot use it for postural control and as a result CNS has to compensate for this loss by relying more on other sources of sensory information, in this case on proprioceptive input from ankle muscles (TS) [5,23]. Similar results have been reported in patients with low back pain $[4,24]$. Reliance on ankle muscles for postural control, known as inverted pendulum model of postural control [25], might be efficient during quiet standing but for more complex tasks, this kind of strategy might result in loss of postural control and even falling.

In the current study, similar to Shanahan et al. [11], no significant differences in response to vibration of VM muscle were found for any of the three groups. A possible explanation of this

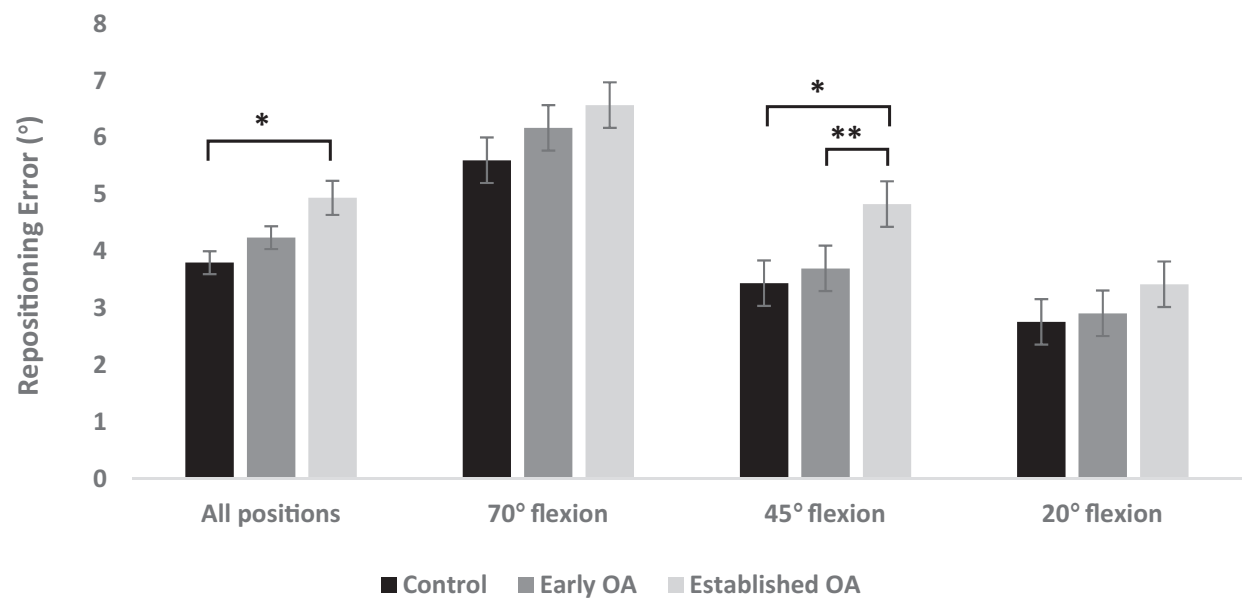

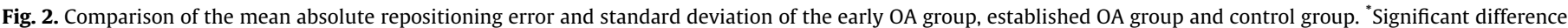

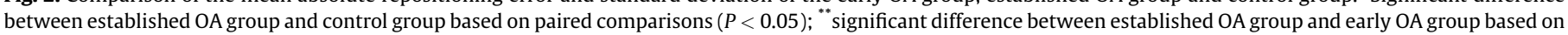
paired comparisons $(P<0.05)$. 
finding might be that the sensory contribution of quadriceps muscle to postural control is limited in the presence of intact sensory information from the TS muscle [26] both in the control and OA participants. But participants with knee OA show a larger response to TS vibration and thus seem to upweight the input from TS for balance control.

Although there was a trend of larger CoP shifts under TS vibration in participants with established OA compared to participants with early $\mathrm{OA}$, we did not find statistically significant differences in vibration responses and in proprioceptive weighting between the two OA groups. Therefore, this might suggest that upweighting of TS information was already present in early stages of knee OA rather than a contributing factor for progression of the disease.

In the present study, an upweighting of TS information was also observed in participants with early knee OA, despite the fact that in this group as opposed to the established OA group, no significant changes in proprioceptive accuracy were measured by the active repositioning test. There were no significant correlations between proprioceptive weighting and repositioning error. Knee joint mechanoreceptors and knee muscle spindles both have major roles in joint position and movement perception $[27,28]$. Knee joint mechanoreceptors are at the primary site of pathology in knee OA and muscle spindles are also known to be altered by knee OA [29,30]. Differences in proprioceptive accuracy as tested with repositioning tests may be explained by differences in the damage to the joint and consequently to the joint mechanoreceptors, which is more severe in established OA compared to the early group. However, the proprioceptive weighting changes observed in the current study already in the early stage of OA, might be more related with movement detection thresholds. This is in agreement with previous findings of increased movement detection thresholds in OA patients irrespective of the stage of the disease and even present in the unaffected knee [7].

A limitation of this study is that all of the participants in the current study were females, and as such the results of this study cannot be generalized to the whole population of patients with knee OA. In addition, postural control in this study was assessed in a static position, so the results cannot be generalized to more dynamic situations. The present study was cross-sectional in nature, considering the progressive nature of the knee $\mathrm{OA}$, it would be useful to investigate the proprioceptive impairments in a longitudinal study.

The results from this study suggest that the early knee OA as well as the established knee OA were associated with upweighting of the proprioceptive information from TS muscle in control of upright stance, which implies an increased reliance on ankle proprioceptive input in both early and established OA groups compared to the asymptomatic controls.

\section{Conflict of interest}

The authors declare that they have no conflicts of interest.

\section{Acknowledgements}

This research was funded by the European Commission through MOVE-AGE, an Erasmus Mundus Joint Doctorate program (20112015). Sjoerd M. Bruijn was supported by a grant from the Netherlands Organization for Scientific Research (NWO \#451-12-041).

\section{References}

[1] Peterka R. Sensorimotor integration in human postural control. J Neurophysiol 2002;88(3):1097-118.
[2] Bloem BR, Allum JHJ, Carpenter MG, Honegger F. Is lower leg proprioception essential for triggering human automatic postural responses? Exp Brain Res 2000;130(3):375-91.

[3] Griffin JW, Cornblath DR, Alexander E, Campbell J, Low PA, Bird S, et al. Ataxic sensory neuropathy and dorsal root ganglionitis associated with Sjögren's syndrome. Ann Neurol 1990;27(3):304-15.

[4] Brumagne S, Cordo P, Verschueren S. Proprioceptive weighting changes in persons with low back pain and elderly persons during upright standing. Neurosci Lett 2004;366(1):63-6.

[5] Horak FB, Shupert CL, Mirka A. Components of postural dyscontrol in the elderly: a review. Neurobiol Aging 1989;10(6):727-38.

[6] Sharma L, Pai YC, Holtkamp K, Rymer WZ. Is knee joint proprioception worse in the arthritic knee versus the unaffected knee in unilateral knee osteoarthritis? Arthritis Rheum 1997;40(8):1518-25.

[7] Koralewicz LM, Engh GA. Comparison of proprioception in arthritic and agematched normal knees. J Bone Joint Surg Am 2000;82(11). 1582-1588.

[8] Baert IA, Mahmoudian A, Nieuwenhuys A, Jonkers I, Staes F, Luyten FP, et al. Proprioceptive accuracy in women with early and established knee osteoarthritis and its relation to functional ability: postural control, and muscle strength. Clin Rheumatol 2013;32(9):1365-74.

[9] Masui T, Hasegawa Y, Yamaguchi J, Kanoh T, Ishiguro N, Suzuki S. Increasing postural sway in rural-community-dwelling elderly persons with knee osteoarthritis. J Orthop Sci 2006;11(4):353-8.

[10] Hinman RS, Bennell KL, Metcalf BR, Crossley KM. Balance impairments in individuals with symptomatic knee osteoarthritis: a comparison with matched controls using clinical tests. Rheumatology 2002;41(12):1388-94.

[11] Shanahan CJ, Wrigley TV, Farrell MJ, Bennell KL, Hodges PW. Postural response to vibration of triceps surae, but not quadriceps muscles, differs between people with and without knee osteoarthritis. J Orthop Res 2014;32(8):989-96.

[12] Brown M, Engberg I, Matthews P. The relative sensitivity to vibration of muscle receptors of the cat. J Physiol 1967;192(3):773-800.

[13] Eklund G. General features of vibration-induced effects on balance. Ups J Med Sci 1972;77(2):112-24.

[14] Kellgren J, Lawrence J. Radiological assessment of osteo-arthrosis. Ann Rheum Dis 1957;16(4):494-502.

[15] Baert IA, Staes F, Truijen S, Mahmoudian A, Noppe N, Vanderschueren G, et al. Weak associations between structural changes on MRI and symptoms, function and muscle strength in relation to knee osteoarthritis. Knee Surg Sports Traumatol Arthrosc 2014;22(9):2013-25.

[16] Hunter DJ, Lo GH, Gale D, Grainger AJ, Guermazi A, Conaghan PG. The reliability of a new scoring system for knee osteoarthritis MRI and the validity of bone marrow lesion assessment: BLOKS (Boston-Leeds Osteoarthritis Knee Score) Ann Rheum Dis 2008;67(2):206-11.

[17] Luyten FP, Denti M, Filardo G, Kon E, Engebretsen L. Definition and classification of early osteoarthritis of the knee. Knee Surg Sports Traumatol Arthrosc 2012:20(3):401-6.

[18] Felson DT, Smolen JS, Wells G, Zhang B, van Tuyl LH, Funovits J, et al. American College of Rheumatology/European League Against Rheumatism provisional definition of remission in rheumatoid arthritis for clinical trials. Arthritis Rheum 2011:63(3):573-86.

[19] Roos EM, Toksvig-Larsen S. Knee injury and Osteoarthritis Outcome Score (KOOS)-validation and comparison to the WOMAC in total knee replacement. Health Qual Life Outcomes 2003;1(1):17.

[20] de Groot IB, Favejee MM, Reijman M, Verhaar JA, Terwee CB, et al. The Dutch version of the Knee Injury and Osteoarthritis Outcome Score: a validation study. Health Oual Life Outcomes 2008:6(1):16.

[21] Roll J, Vedel J. Kinaesthetic role of muscle afferents in man: studied by tendon vibration and microneurography. Exp Brain Res 1982;47(2):177-90.

[22] Bennell KL, Hinman RS, Metcalf BR, Crossley KM, Buchbinder R, Smith M, et al. Relationship of knee joint proprioception to pain and disability in individuals with knee osteoarthritis. J Orthop Res 2003;21(5):792-7.

[23] Carver S, Kiemel T, Jeka JJ. Modeling the dynamics of sensory reweighting. Biol Cybern 2006;95(2):123-34

[24] Brumagne S, Janssens L, Knapen S, Claeys K, Suuden-Johanson E. Persons with recurrent low back pain exhibit a rigid postural control strategy. Eur Spine J 2008;17(9):1177-84.

[25] Winter DA, Patla AE, Prince F, Ishac M, Gielo-Perczak K. Stiffness control of balance in quiet standing. J Neurophysiol 1998;80(3):1211-21.

[26] Hirata RP, Arendt-Nielsen L, Shiozawa S, Graven-Nielsen T. Experimental knee pain impairs postural stability during quiet stance but not after perturbations. Eur J Appl Physiol 2012:112(7):2511-21.

[27] Inglis J, Frank J, Inglis B. The effect of muscle vibration on human position sense during movements controlled by lengthening muscle contraction. Exp Brain Res 1991;84(3):631-4.

[28] Proske U, Gandevia SC. The kinaesthetic senses. J Physiol 2009;587(17):4139 46.

[29] Ikeda S, Tsumura H, Torisu T. Age-related quadriceps-dominant muscle atrophy and incident radiographic knee osteoarthritis. J Orthop Sci 2005;10(2): 121-6.

[30] Petterson SC, Barrance P, Buchanan T, Binder-Macleod S, Snyder-Mackler L. Mechanisms underlying quadriceps weakness in knee osteoarthritis. Med Sci Sports Exerc 2008;40(3):422. 